\title{
LOS VICIOS DE ORIGEN DE LOS PROCESOS AUTONÓMICOS
}

\author{
CARLOS RUIZ MIGUEL \\ Catedrático de Derecho Constitucional \\ Universidad de Santiago de Compostela
}

\author{
SUMARIO \\ I. Propósito de este trabajo. \\ II. Los vicios de origen del primer proceso \\ autonómico. \\ III. Los vicios de origen del segundo proce- \\ so autonómico. \\ IV. Conclusión.
}

\section{PROPÓSITO DE ESTE TRABAJO.}

La aprobación del nuevo Estatuto de Cataluña y el proceso estatutario desencadenado para acompañarlo han supuesto la mayor crisis, quizá terminal, sufrida por el sistema constitucional inaugurado en 1978.

En este trabajo me propongo argumentar que tanto el primer como el segundo proceso estatutario padecen graves vicios de origen que le privan de legitimidad.

Sugeriré que las semillas de la destrucción (o "deconstrucción") del sistema autonómico se plantaron ya en 1979, en el primer año de vigencia de la Constitución de 1978 cuando se desarrolló por primera vez el proceso estatutario sin respetar el texto de la Constitución que se acababa de adoptar. El problema se agravó cuando esas semillas se pudieron desarrollar porque el Tribunal Constitucional, lejos de arrancarlas, permitió que crecieran. Esas semillas eran procedimentales y sustanciales, pero en este trabajo estudiaremos sólo las primeras.

Aquellos precedentes alentaron el desencadenamiento de un segundo proceso autonómico, vertebrado en torno al nuevo Estatuto de Cataluña, donde se reiteró la falta de respeto a las previsiones constitucionales de tipo formal y se llevó al paroxismo la vulneración de previsiones constitucionales 
sustantivas. La falta de cumplimiento por el TC de su misión originaria de respeto a la Constitución en relación con el primer proceso estatutario se convierte ahora en un peligroso precedente de lo que puede ocurrir en el segundo proceso estatutario.

\section{LOS VICIOS DE ORIGEN DEL PRIMER PROCESO AUTONÓMICO}

\section{II.1. RefERÉndums CONVOCAdOS InCONSTITUCIONALMENTE: País Vasco y Cataluña}

II.1.A. La Constitución establece que, en el supuesto del acceso privile-

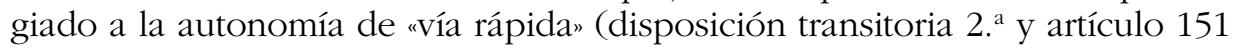
CE), una vez que la propuesta de Estatuto por la asamblea de diputados y senadores de las provincias interesadas haya sido aceptada por la Comisión Constitucional del Congreso, se deberá celebrar un referéndum de ratificación de la iniciativa antes de que la misma sea aprobada por las Cortes Generales y posteriormente sancionada y promulgada como ley orgánica (art. 151.2.3. $\left.{ }^{\circ} \mathrm{CE}\right)$.

La Constitución establece en su art. 92.3 CE que «Una Ley orgánica regulará las condiciones y el procedimiento de las distintas modalidades de referéndum previstas en esta Constitución". Esta materia, por tanto, está expresamente reservada al instrumento "ley orgánica".

La Constitución, por otro lado, en el art. 86.1, establece que el DecretoLey que procede en situaciones de extraordinaria y urgente necesidad, no puede afectar al "ordenamiento de las instituciones básicas del Estado, ..., al régimen de las Comunidades Autónomas, ni al derecho electoral general". Parece claro que un Estatuto de autonomía afecta a las instituciones básicas del Estado, al régimen de las Comunidades Autónomas y al derecho electoral en cuanto a los requisitos establecidos para considerar válido un resultado referendario.

El art. 92.3 CE fue desarrollado por la Ley Orgánica 2/1980, de 18 de enero, sobre Regulación de las Distintas Modalidades de Referéndum, que en su art. 8 regula ratificación por referéndum de la iniciativa autonómica prevista en el artículo 151.1 de la Constitución y sólo cuatro meses después de los Reales Decretos convocando los referenda.

II.1.B. Para la elaboración de los Estatutos de autonomía del País Vasco y Cataluña hubo tantas prisas que ni siquiera se esperó a la aprobación de la Ley Orgánica 2/1980, que fue aprobada a poco más de un año de la entrada en vigor de la Constitución.

En efecto, el Gobierno aprobó, y el Congreso de los Diputados convalidó, sendos Reales Decretos-Leyes para convocar los referéndums de iniciativa autonómica del País Vasco y Cataluña, pese a que aún no se había aprobado la ley orgánica que debía regular estos referenda. 
El texto del Real Decreto-Ley 13/1979, de 14 de septiembre, por el que se somete a referéndum el Proyecto de Estatuto de Autonomía para el País Vasco y del Real Decreto-Ley 14/1979, de 14 de septiembre, por el que se somete a referéndum el Proyecto de Estatuto de Autonomía de Cataluña ${ }^{1}$ es similar, dejando al margen la reproducción del texto de los respectivos Estatutos en sus respectivas disposiciones adicionales.

Pero lo importante es que la propia Exposición de motivos de ambos Reales Decretos-Leyes confiesa que hay "inexistencia de normas de desarrollo de la Constitución relativas al referéndum". Ello no obstó a que el Congreso de los Diputados convalidara ambos Reales Decretos-Leyes, en sendas resoluciones de 27 de septiembre de $1979^{2}$.

I.1.C. A la luz de lo expuesto, resulta, de todo punto evidente, que en la aprobación de los Estatutos de autonomía del País Vasco y de Cataluña-1979 se vulneraron los arts. 81, 86.1 y 92.1 de la Constitución.

\section{II.2. Renegociación InCONSTItUCiOnAL: Galicia}

II.2.A. La Constitución establece que, en la tramitación de las iniciativas de acceso a la autonomía de "Vía rápida", "Aprobado el proyecto de Estatuto por la Asamblea de Parlamentarios, se remitirá a la Comisión Constitucional del Congreso, la cual, dentro del plazo de dos meses, lo examinará con el concurso y asistencia de una delegación de la Asamblea proponente para determinar de común acuerdo su formulación definitiva" (art. 151.2.2 CE).

La posición de la Comisión Constitucional del Congreso no es de «igual a igual" con la de la Asamblea de Parlamentarios. En efecto, mientras que la Comisión Constitucional puede aprobar cuantos cambios desee en el proyecto de Estatuto, no puede hacer lo mismo la "Asamblea de parlamentarios" redactora de la iniciativa.

Las consecuencias son que, "si se alcanzare dicho acuerdo (entre la Comisión Constitucional del Congreso y la "Asamblea de Parlamentarios" redactora de la iniciativa), el texto resultante será sometido a referéndum del cuerpo electoral de las provincias comprendidas en el ámbito territorial del proyectado Estatuto" (art. 151.2.3 CE). Ahora bien, "de no alcanzarse el acuerdo a que se refiere el apartado 2 de este número, el proyecto de Estatuto será tramitado como proyecto de Ley ante las Cortes Generales" (art. 151.2.5 CE).

La diferencia entre ambos supuestos está clara. En el primer caso (art. 151.2.3 CE), una vez que se produce el acuerdo entre «Comisión Constitucional" y "Asamblea de parlamentarios" el "texto resultante" "será sometido a referéndum. Si el voto en referéndum es positivo, las Cortes Generales simplemente ratificarán o rechazarán la iniciativa. En el segundo caso, si no hay

1 Publicados ambos en el Boletín Oficial del Estado de 24 de septiembre de 1979.

2 Publicadas ambas en el Boletín Oficial del Estado de 10 de octubre de 1979 
acuerdo entre Comisión Constitucional y "Asamblea de Parlamentarios", la iniciativa de ésta se tramitará como "proyecto de ley", lo que significa que las Cortes Generales tienen plena capacidad de enmienda sobre el texto presentado por la "Asamblea de parlamentarios". Será el texto tal y como lo hayan aprobado las Cortes Generales el que será sometido a referéndum y, posteriormente, promulgado.

II.2.B. En el caso de Galicia, la "Asamblea de Parlamentarios" presentó su propuesta de Estatuto al Presidente del Congreso el 28 de junio de 1979, siendo publicada en el Boletín Oficial de las Cortes el 20 de julio de 1979. El 10 de septiembre se publicaba el acuerdo de remitir el texto de la "Asamblea de Parlamentarios" a la Comisión Constitucional del Congreso. La ponencia de la Comisión Constitucional examinó el texto y aprobó un Infor$\mathrm{me}^{3}$. Ese Informe fue discutido en la Comisión Constitucional con la asistencia de la "Asamblea de Parlamentarios" los días 20 y 21 de noviembre de 1979 y fue aprobado. El Dictamen de la Comisión, elaborado «de común acuerdo" con la "Asamblea de Parlamentarios incluyó la introducción de un apartado 2 de la disposición $3 .^{\text {a }}$ del proyecto de Estatuto que no figuraba en el Informe de la Ponencia. El Dictamen de la Comisión aprobado de común acuerdo con la "Asamblea de Parlamentarios" se publicó oficialmente ${ }^{4}$.

De acuerdo con lo previsto en el art. 151.2.3 CE "si se alcanzare dicho acuerdo (entre la Comisión Constitucional del Congreso y la "Asamblea de Parlamentarios" redactora de la iniciativa), el texto resultante será sometido a referéndum del cuerpo electoral de las provincias comprendidas en el ámbito territorial del proyectado Estatuto".

Sin embargo, el texto no se sometió a referéndum. Y no fue así por la presión política de los partidos distintos de la Unión de Centro Democrático que sostenían que el Estatuto debía aprobarse por "consenso" y al no existir tal "consenso" sino "mayoría" rechazaron el Proyecto.

El Presidente de la "Asamblea de Parlamentarios de Galicia" remitió a la presidencia del Congreso de los Diputados un "escrito" aprobado por la citada "Asamblea" el 3 de octubre de 1980 en relación con el proyecto de Estatuto5. En ese escrito, se dice, textualmente que

"El 22 de noviembre de 1979 fue aprobado por acuerdo mayoritario de la Comisión Constitucional y la Delegación de la Asamblea de Parlamentarios de Galicia un texto de Estatuto de Autonomía para Galicia.

El acuerdo, aunque válido desde el punto de vista jurídico-constitucional, resulta políticamente insuficiente para que el futuro Estatuto obtenga, en el Referéndum correspondiente, un respaldo ampliamente mayoritario de las fuerzas políticas, para conseguir se constituya, como norma fundamental para la convivencia y el entendimiento de los gallegos y no para su división o enfrentamiento (...).

3 Publicado en el Boletín Oficial de las Cortes Generales de 15 de noviembre de 1979.

4 En el Boletin Oficial de las Cortes Generales de 14 de diciembre de 1979.

5 Publicado en el Boletín Oficial de las Cortes Generales el 28 de octubre de 1980. Este escrito se publicó en la serie $\mathrm{H}$ "Otros textos normativos" 
En consecuencia, los partidos políticos firmantes, acuerdan solicitar del Presidente de la Xunta de Galicia la convocatoria de la Asamblea de Parlamentarios de Galicia para que, asumiendo, en su caso, este acuerdo, pueda dirigirse al Excmo. Sr. Presidente del Congreso de los Diputados, a fin de que, por el órgano que corresponda y con los asesoramientos que se estimen pertinentes, se sirva convocar a la Comisión Constitucional y a la Delegación de la Asamblea de Parlamentarios de Galicia para llevar a cabo, antes de la convocatoria de Referéndum, la modificación, si procede, de los puntos siguientes, complementando así la acción realizada en noviembre pasado (...).

Recibido este escrito, se produce el escándalo de que la Comisión Constitucional apruebe el 22 de octubre de 1980 un "acuerdo" por el que accede a lo solicitado ${ }^{6}$. En ese acuerdo se dice lo siguiente:

"La Comisión Constitucional, en su sesión del día de hoy, ha adoptado, en relación con el escrito formulado por el Presidente de la Asamblea de Parlamentarios de Galicia, con fecha 3 de octubre de 1980, el siguiente acuerdo:

"1. Aceptar, con voluntad conceder a la Asamblea de Parlamentarios de Galicia, la reconsideración de los aspectos concretos del proyecto de Estatuto de Autonomía a que taxativamente se refiere el escrito del Presidente de dicha Asamblea.

"2. Comunicar a la Presidencia del Congreso el resultado para que en su caso lo comunique al Gobierno a los efectos de la suspensión del trámite de referéndum pendiente."

El mismo día (4-XI-1980) en que se publica el "escrito" aceptando reconsiderar el proyecto válidamente aprobado desde el punto de vista jurídicoconstitucional, se publica también en el Boletín Oficial de las Cortes Generales el nuevo proyecto de autonomía de Galicia que recoge los puntos presentados por la "Asamblea de Parlamentarios".

II.2.C. Es evidente que en la tramitación del Estatuto de Galicia se ha violado el art. 151.2.3 CE pues, una vez aprobado el Dictamen de la Ponencia con el concurso y el acuerdo de la "Asamblea de Parlamentarios", la Constitución prescribía que "el texto resultante será sometido a referéndum".

Antes bien, el texto resultante del acuerdo alcanzado no se sometió a referéndum y lo que se sometió a referéndum fue la revisión de un texto ya aprobado, lo cual es totalmente contrario a la Constitución. Un referéndum en el que conviene recordarlo, participó sólo el 28,27\% de censo electoral y donde entre quienes acudieron a votar, hubo un 19,77\% de votos en contra, un $4,62 \%$ de votos en blanco y un $2,25 \%$ de votos nulos ${ }^{7}$.

6 Publicado en el Boletín Oficial de las Cortes Generales el 4 de noviembre de 1980. Este escrito se publicó en la serie $\mathrm{H}$ "Otros textos normativos"

7 De un total de 2.172.898 electores, participaron 614.218. De entre los votantes, 450.556 lo hicieron a favor, 121.448 en contra, optando por el blanco 28.381 y haciéndolo de forma nula 18.383. Cfr: http://narros.congreso.es/constitucion/elecciones/referendos/ref_gali.htm 


\section{II.3. VACIAMIENTO INCONSTITUCIONAL DEL RESUlTADO DE UN REFERÉNDUM: AndaluCía}

II.3.A. El acceso a la autonomía por la "Vía rápida" está en la Constitución abierto a todas las regiones. Ahora bien, aquellas que no gozaban del privilegio dispuesto en la disposición transitoria $2 .{ }^{a} \mathrm{CE}$, tenían que seguir un proceso ciertamente dificultoso previsto en el art. 151.1 CE. Este precepto exigía la concurrencia de los siguientes requisitos para acceder a la autonomía por vía rápida: a) que en un plazo de 6 meses todas las Diputaciones provinciales de la región suscriban la iniciativa; b) que en un plazo de 6 meses se sumen a la iniciativa los Municipios de de cada una de las provincias afectadas que representen, al menos, la mayoría del censo electoral de cada una de ellas; y c) que "dicha iniciativa sea ratificada mediante referéndum por el voto afirmativo de la mayoría absoluta de los electores de cada provincia, en los términos que establezca una Ley Orgánica".

II.3.B. En el caso de Andalucía, ese referéndum se convocó mediante Real Decreto 145/1980, de 26 de enero para ser celebrado el 28 de febrero de 1980. En dicho referéndum, en la provincia de Almería la iniciativa obtuvo el voto afirmativo del $42,31 \%$ de los electores ${ }^{8}$. Esto significa, pura y sencillamente que no se dio el supuesto de hecho previsto en el art. 151.1 CE para que la iniciativa pudiera seguir adelante.

Sin embargo, tras este resultado, se aprobaron las Leyes Orgánicas 12 y 13/1980, ambas de 16 de diciembre, para permitir que Andalucía siguiera adelante con la iniciativa autonómica por la "vía rápida".

La primera de esas normas, la LO 12/1980, de 16 de diciembre, de modificación del párrafo cuarto del artículo octavo de la Ley Orgánica reguladora de las distintas modalidades de referéndum, introdujo una disposición según la cual:

«a iniciativa autonómica prevista en el artículo ciento cincuenta y uno se entenderá ratificada en las provincias en las que se hubiere obtenido la mayoría de votos afirmativos previstos en el párrafo anterior, siempre y cuando los votos afirmativos hayan alcanzado la mayoría absoluta del censo de electores en el conjunto del ámbito territorial que pretenda acceder al autogobierno.

Previa solicitud de la mayoría de los Diputados y Senadores de la provincia o provincias en las que no se hubiera obtenido la ratificación de la iniciativa, las Cortes Generales, mediante Ley Orgánica, podrán sustituir la iniciativa autonómica prevista en el artículo ciento cincuenta y uno siempre que concurran los requisitos previstos en el párrafo anterior”.

A continuación, el mismo día, en aplicación de esta ley se aprobó la LO 13/1980, de 16 de diciembre, de sustitución en la provincia de Almería de la iniciativa autonómica que, en su artículo único decía que:

8 En la provincia de Alméria el número de electores era de 279.300 y el de votantes de 142.769, de los cuales votaron sí a la iniciativa 118.186 (es decir, el 42,31\% de los electores). Ver los resultados en: http://narros.congreso.es/constitucion/elecciones/referendos/ref_and_80.htm 
"Habiéndose producido la solicitud de los Diputados y Senadores de la provincia de Almería, a la que alude la Ley Orgánica sobre la regulación de las distintas modalidades de referéndum, las cortes generales, por los motivos de interés nacional a los que se refiere el título VIII de la Constitución, declaran sustituida en esta provincia la iniciativa autonómica con objeto de que se incorpore al proceso autonómico de las otras provincias andaluzas por el procedimiento del artículo 151 de la Constitución".

II.3.C. Es evidente que lo que dispone la LO 12/1980 es radicalmente contrario a lo que prescribe el art. 151.1 CE. Por ello, la aplicación que hace la LO 13/1980 está igualmente aquejada de inconstitucionalidad. La doctrina española en este punto es clara, aunque algunos autores utilicen eufemismos para calificar este hecho?.

Sin consideración a este vicio, el procedimiento autonómico siguió su curso y el RD 1835/1981, de 20 de agosto, convocó un referéndum para la aprobación del proyecto de Estatuto de Autonomía. Este referéndum se celebró el 20 de octubre de 1981.

\section{LOS VICIOS DE ORIGEN DEL SEGUNDO PROCESO AUTONÓMICO}

\section{III.1. LA INCONSTITUCIONAL APROBACIÓN DE NUEVOS ESTATUTOS COMO REFORMAS}

\section{III.1.A. La elemental distinción entre reforma y derogación novativa.}

III.1.A.a. Un conocimiento elemental de la teoría de la norma jurídica evidencia que una cosa es "modificar" una ley y otra hacer una ley "nueva". En efecto, se denominan "leyes nuevas" a las que "regulan por primera vez una determinada materia" y a las que "contienen una nueva redacción de otra ley anterior", mientras que son "leyes modificativas" las que contienen "una nueva redacción de partes de una ley anterior", las que "añaden disposiciones nuevas o suprimen algunas de las existentes" y las que "prorrogan o suspenden la vigencia de una ley" ${ }^{10}$. Cuando se elabora una "ley modificativa", la técnica legislativa correcta exige fórmulas como:

9 Así, por ejemplo, un especialista en materia de referéndum habla del "rocambolesco proceso seguido para crear la Comunidad Autónoma de Andalucía" donde la reforma de la LO 2/1980 permitió "trastocar" los resultados del referéndum. Cfr. L. AGUIAR DE LUQUE, "Referéndum", en M. ARAGÓN REYES (coord.), Temas básicos de Derecho Constitucional, t. I, Civitas, Madrid, 2001, págs. 125 ss.

10 M. T. CASTIÑEIRA PALOP, "Leyes modificativas", en VV.AA., La función legislativa de los parlamentos y la técnica de legislar. III Jornadas de Derecho Parlamentario, Congreso de los Diputados, Madrid, 2000, pág. 73. En el mismo sentido, M. MARTÍN CASALS, "La estructura básica de un manual español de técnica legislativa", pág. 263, en A. Menéndez Menéndez (Dir.) y A. PAU PEDRÓN (director adjunto), La proliferación legislativa: un desafio para el Estado de Derecho, Thomson-Civitas/Colegio Libre de Eméritos, Madrid, 2004. 
«Ley Orgánica 6/1987, de 11 de noviembre por la que se modifica la sección del capítulo 40 del Código Penal.

Artículo 1.

El artículo 534 del Código Penal tendrá en lo sucesivo la redacción que se inserta a continuación.....11.

Como es obvio, esta diferenciación entre la ley "nueva" y la ley "modificativa" no es una rareza de nuestro sistema jurídica, sino que existe en los demás ordenamientos jurídicos. Así ocurre en los Estados Unidos de América ${ }^{12}$ o en Alemania ${ }^{13}$.

III.1.A.b. A la luz de estas nociones elementales, cabe analizar formalmente los diversos cambios que se están experimentando en los Estatutos de autonomía. Conviene advertir, por último, que la "reforma de los Estatutos de autonomía" no ha sido, ni mucho menos, una idea novedosa. La evolución legislativa hasta 2004 muestra que ha habido numerosas reformas que han sido, rigurosamente, eso, reformas, es decir, modificaciones de un texto cuyo vigencia subsitía y no era derogada. No cabe, por tanto, alegar ignorancia o falta de precedentes de lo que significa una operación de "reforma estatutaria".

III.1.B. El precedente: el "Plan Ibarreche»

III.1.B.a. El llamado "Plan Ibarreche» (Propuesta de Estatuto Político de la Comunidad de Euskadi") fue elevado por el Parlamento de la Comunidad Autónoma Vasca a las Cortes Generales para ser tramitado como una "proposición de ley".

Se ha discutido la cuestión sustantiva de cuál es la verdadera de este texto: si es legal o constitucional ${ }^{14}$, pero en este trabajo nos vamos a centrar no en su sustancia, sino en su análisis formal.

El texto clave para este análisis es la disposición final primera del «Plan Ibarreche" que dice lo siguiente:

«... el presente Estatuto político sucederá y sustituirá, a su entrada en vigor, al que fuera aprobado por la Ley Orgánica 3/1979, de 18 de diciembre, de Estatuto de Autonomía para el País Vasco».

11 CASTIÑEIRA PALOP, ibid.

12 J.A. RUIZ GARCÍA, "Técnica legislativa en EE.UU.", en MÉNENDEZ y PAU, cit. pág. 411.

13 Í. ORTIZ DE URBINA GIMENO, "Técnica legislativa en Alemania”, en Menéndez y Pau, cit., págs 448-450

$14 \mathrm{He}$ argumentado la naturaleza constitucional de este texto en "El Estado autonómico y sus enemigos", en La Ley n. 5911 (11-XII-2003), págs. 1-6, posteriormente ampliado en "El Estado autonómico y sus enemigos", en M.A. GARCÍA HERRERA (Ed.), Constitución y democracia. 25 años de Constitución democrática en España, CEPC/Universidad del País Vasco, Bilbao, 2005, vol. II, págs. 475-490, trabajo a su vez desarrollado en "La indefensión jurisdiccional del poder constituyente y la destrucción de la Constitución", en E. FERRER MC-GREGOR y A. ZALDÍVAR LELO DE LARREA (Coords.), La Ciencia del Derecho Constitucional. Homenaje a Héctor FixZamudio en sus cincuenta años como investigador del Derecho, UNAM/Instituto Mexicano de Derecho Procesal Constitucional/Marcial Pons, México, 2008, tomo III, pág. 515-551. 
Esta disposición deja claro que el llamado "Plan Ibarreche" no era una reforma sino una derogación expresa de todo el Estatuto de Autonomía vigente $^{15}$. No admite discusión, por tanto, que este texto era cualquier cosa menos una "reforma estatutaria".

III.1.B.b. La Mesa del Congreso de los Diputados publicó la iniciativa en el Boletín Oficial de las Cortes Generales. Congreso de los Diputados Núm. B-149-1 de 21/01/2005 págs. 1 y ss. Esta publicación se hizo dentro de la "Serie B: PROPOSICIONES DE LEY". Insisto en que en este lugar no voy a reproducir una argumentación realizada en otro lugar negando que la naturaleza del texto sea la de una "ley".

Ahora bien, sin entrar a discutir la cuestión sustancial, conviene denunciar que la Mesa del Congreso en su reunión de 21 de enero de 2005 acordó admitir a trámite el "plan Ibarretxe" y lo calificó como "Propuesta de reforma de Estatuto de Autonomían. Para ello adujo unos supuestos fundamentos jurídicos totalmente fraudulentos. La resolución de la Mesa decía dictarse "Al amparo de lo dispuesto en el artículo 31.1.4.. ${ }^{\circ}$ del Reglamento" ${ }^{16}$ y "de conformidad con el punto séptimo de la Resolución de la Presidencia del Congreso de los Diputados sobre procedimiento a seguir para la tramitación de la reforma de los Estatutos de Autonomía, de 16 de marzo de 1993".

A nuestro juicio, es evidente que esta calificación por la Mesa del llamado "Plan Ibarreche" como un caso "reforma de los Estatuto de Autonomía", es un notorio fraude de la normativa vigente, porque, como hemos indicado, la disposición final primera de esa propuesta es inequívoca al indicar que no trataba de "reformar" el Estatuto de Guernica de 1979, sino de "sucederlo y sustituirlo". No era, por tanto, una "reforma" estatutaria.

III.2.B.c. Esta iniciativa fue rechazada tras el debate de toma en consideración y no llegó a entrar en vigor. Pero este hecho puede ocultar la verdadera trascendencia formal de lo ocurrido. Y lo que ocurrió fue, nada más y nada menos, que la Mesa del Congreso calificó como una «reforma estatutaria" a algo que no lo era. Se creaba así el precedente para dar luz verde a la tramitación como "reformas" estatutarias de textos que no eran tales "reformas" sino textos que liquidaban los Estatutos aprobados en la transición.

III.1.C. La consumación: Cataluña, Andalucía, Aragón.

Una vez sentado por la Mesa del Congreso el precedente de considerar como "reforma estatutaria" (el llamado "Plan Ibarreche") lo que no era "reforma" ni tampoco, a mi juicio, "estatutaria", se dejó abierta la puerta para utilizar este precedente en otros casos. Y así ocurrió.

III.1.C.a. El primer supuesto fue el caso de Cataluña. El Parlamento regional elegido en 2003 decidió impulsar un nuevo Estatuto de autonomía que

15 «...el presente Estatuto político sucederá y sustituirá, a su entrada en vigor, al que fuera aprobado por la Ley Orgánica 3/1979, de 18 de diciembre, de Estatuto de Autonomía para el País Vasco".

16 Este precepto dice que "Corresponden a la Mesa las siguientes funciones: $4 .^{\circ}$ Calificar, con arreglo al Reglamento, los escritos y documentos de indole parlamentaria, así como declarar la admisibilidad o inadmisibilidad de los mismos." 
sustituyera al vigente, aprobado en 1979 que, como hemos visto, tuvo tachas de ilegitimidad de origen. El Parlamento de Cataluña tramitó una "Propuesta de Proposición de Ley orgánica por la que se establece el Estatuto de autonomía de Cataluña y se deroga (cursiva mía) la Ley orgánica 4/1979, del 18 de diciembre, del Estatuto de autonomía de Cataluña (en lo sucesivo Propuesta de Reforma - cursiva mía-)". Ante esta denominación sólo caben dos opciones: o el Parlamento de Cataluña ignora la distinción elemental entre "derogación" y "reforma", o buscaba deliberadamente un fraude constitucional. De lo que no cabía ninguna duda es de que la proposición incluía una disposición derogatoria del Estatuto de 1979. Por eso mismo, resultó asombroso ver cómo profesores de Derecho Constitucional podían hablar de "legitimidad" de la "reforma" estatutaria catalana ${ }^{17}$.

El Consejo Consultivo catalán, competente para conocer sobre "proposiciones de leyes del Parlamento de Cataluña" y velar por la "estatutoriedad" de las leyes "del Parlamento de Cataluña", no tuvo empacho en declararse competente para conocer de una iniciativa que ni era una proposición de "ley del Parlamento de Cataluña" (puesto que era una proposición de ley orgánica de las Cortes Generales"), ni respetaba el Estatuto (puesto que se proponía derogarlo) ${ }^{18}$. Esto resulta especialmente llamativo porque el Consejo Consultivo, como he dicho, no se cuestiona en ningún momento si es posible derogar el Estatuto de 1979, a pesar de que fue creado para velar por el respeto al Estatuto de 1979.

De nada serviría, para justificar que es una "reforma", el hecho de que en su elaboración se halla seguido el procedimiento de reforma del Estatuto previsto en el propio Estatuto (Ley Orgánica 4/1979). Tal argumento es insostenible pues la operación de utilizar una norma (las normas sobre "reforma" del Estatuto) para obtener un objetivo contrario al previsto (es decir, para no reformar, sino para liquidar el Estatuto) constituye un fraude de ley. En consecuencia, la aprobación de un texto sustancialmente nuevo no queda legitimada por el hecho de que se haga utilizando la norma prevista para "reformar" un texto anterior. La conclusión contraria llevaría a conclusiones absurdas y disparatadas. En efecto, de aceptar tales argumentos nada impediría que, siguiendo el procedimiento de "reforma estatutaria" el Parlamento de Cataluña aprobara una Constitución para Cataluña o aprobase una ley declarando la independencia del territorio.

17 En el breve "Manifiesto de Profesores españoles de Derecho Constitucional" [cfr. http:// www.iceta.org/2005/11/un-important-manifest-ha-passat.htm firmado por varios catedráticos y profesores titulares de Derecho Constitucional, se encuentran estos tres pasajes: "aprobación por el Parlamento de Cataluña de un proyecto de reforma estatutaria"; "derecho que asiste al Parlamento de Cataluña para ejercer la iniciativa de reforma de un Estatuto", "el proceso de reforma estatutaria iniciado en Cataluña y en otras Comunidades Autónomas..." (cursivas mías). No deja de ser sorprendente que en este manifiesto se diga (con razón) que el plan Ibarreche "no supone una reforma del Estatuto de Guernika" (p. 517)

18 Dictamen 269/2005, de 1 de septiembre, del Consejo Consultivo de Cataluña, sobre la Propuesta de Proposición de Ley Orgánica por la que se establece el Estatuto de Autonomía de Cataluña y se deroga la Ley Orgánica 4/1979, de 18 de diciembre, del Estatuto de autonomía de Cataluña. 
Una vez aprobado por el Parlamento catalán la propuesta de nuevo Estatuto que pretendía derogar el Estatuto de 1979, se remitió al Congreso de los Diputados. La Mesa del Congreso, mediante acuerdo de 18 de octubre de 2005, decidió "Al amparo de lo dispuesto en el artículo 31.1.4. del Reglamento" "19, "Calificar la iniciativa como Propuesta de reforma (cursiva mía) de Estatuto de Autonomía ${ }^{20}$. La propuesta siguió la tramitación prevista en la Resolución de la Presidencia del Congreso de los Diputados sobre procedimiento a seguir para la tramitación de la reforma de los Estatutos de Autonomía, de 16 de marzo de 1993. Finalmente el texto se aprobó como Ley Orgánica 6/2006, de 19 de julio, de reforma del Estatuto de Autonomía de Cataluña. A pesar de ser denominada como de "reforma", esta ley contiene la siguiente disposición derogatoria:

"Queda derogada la Ley Orgánica 4/1979, de 18 de diciembre, de Estatuto de Autonomía de Cataluña».

En consecuencia, no parece que sea correcto hablar de "reforma" estatutaria, aunque algún autor lo haga ${ }^{21}$.

II.1.C.b. El segundo caso de pseudo-reforma estatutaria es el de Andalucía. Se trata de un caso con muchísimas similitudes con el caso catalán. El Parlamento andaluz promovió una "Proposición de Reforma (cursivas mías) del Estatuto de Autonomía para Andalucía". Esta proposición de "reforma", sin embargo, igual que la catalana, contenía una disposición derogatoria del Estatuto vigente, el de 1981. Como en el caso catalán, la proposición de "reforma" fue objeto de un dictamen por el Consejo Consultivo andaluz ${ }^{22}$. En este dictamen el Consejo dice (FJ II.1) :

al versar la consulta sobre una Proposición de Reforma Estatutaria, no cabe obviamente un examen de legalidad, ni tampoco existen parámetros estatutarios que observar (salvo los preceptos que tienen que ver con el procedimiento de reforma), pues lo que se reforma es justamente el Estatuto de Autonomía. Por ello, el presente dictamen tiene como objetivo básico e ineludible la verificación de la constitucionalidad de las disposiciones contenidas en dicha propuesta, puesto que como ha sentado el Tribunal Constitucional en su sentencia 99/1986, de 11 de julio, el único parámetro para enjuiciar la validez constitucional de una disposición incluida en un Estatuto de Autonomía es la propia Constitución.”

Si el Consejo andaluz tenía como misión velar por el cumplimiento, no sólo de la "Constitución", sino también del "Estatuto de Autonomía" (art. 1. de

19 Ese precepto dice: "Calificar, con arreglo al Reglamento, los escritos y documentos de índole parlamentaria, así como declarar la admisibilidad o inadmisibilidad de los mismos"

20 Acuerdo publicado en el Boletín Oficial de las Cortes Generales, serie B n. ${ }^{\circ} 210-1$, de 21 de octubre de 2005.

21 Se habla de "Vía catalana de reforma estatutaria" (cursiva mía) en algún manual (Enrique ÁlVAREZ CONDE, Curso de Derecho Constitucional, vol. II, Tecnos, Madrid, 2005, pág. 520-521).

22 Dictamen 72/2006, de 10 de marzo, del Consejo Consultivo de Andalucía Proposición de Reforma (sic) del Estatuto de Autonomía para Andalucía. 
la Ley 8/1993, del Consejo Consultivo de Andalucía), debía verificar si la iniciativa sometida a su consideración era verdaderamente una "reforma" que, por tanto, respetaba la esencia del "Estatuto de autonomía" que, reformado, debía subsistir. No lo hizo así el Consejo Andaluz.

Como en el caso catalán y el precedente primero (el "Plan Ibarreche"), esta iniciativa fue calificada por la Mesa del Congreso como "reforma estatutaria" en su acuerdo de 9 de mayo de $2006^{23}$. Como en el caso catalán, siguió la tramitación prevista en la Resolución de la Presidencia del Congreso de los Diputados sobre procedimiento a seguir para la tramitación de la reforma de los Estatutos de Autonomía, de 16 de marzo de 1993. Finalmente el texto se aprobó como Ley Orgánica 2/2007, de 19 de marzo, de reforma del Estatuto de autonomía de Andalucía. A pesar de ser denominada como de "reforma", esta ley contiene la siguiente disposición derogatoria:

“Disposición derogatoria. Derogación de la Ley Orgánica 6/1981, de 30 de diciembre.

Queda derogada la Ley Orgánica 6/1981, de 30 de diciembre, de Estatuto de Autonomía para Andalucía."

III.1.C.c. El tercer caso es el de Aragón. Como ocurrió en los casos de Cataluña y de Andalucía, aquí el Parlamento aragonés elaboró una propuesta de nuevo Estatuto que en su título incluía el nombre de "reforma" pero cuya naturaleza novativa quedaba clara al contener una disposición derogatoria del Estatuto vigente. A diferencia de Cataluña y de Andalucía, aquí no hubo dictamen sobre el texto por parte del Consejo consultivo autonómico aragonés (la Comisión Jurídica Asesora, en ese momento).

Igual que en el caso del "plan Ibarreche" y de las iniciativas de los Parlamentos catalán y andaluz, la iniciativa de las Cortes de Aragón fue calificada por la Mesa del Congreso, en su acuerdo de 5 de septiembre de 2006, como "Propuesta de reforma del Estatuto de autonomía" ${ }^{24}$.

Como en los casos catalán y andaluz, siguió la tramitación prevista en la Resolución de la Presidencia del Congreso de los Diputados sobre procedimiento a seguir para la tramitación de la reforma de los Estatutos de Autonomía, de 16 de marzo de 1993.

Finalmente el texto se aprobó como Ley Orgánica 5/2007, de 20 de abril, de reforma del Estatuto de autonomía de Aragón. A pesar de ser denominada como de "reforma", esta ley contiene la siguiente disposición derogatoria:

¿Disposición derogatoria.

Queda derogada la la Ley Orgánica 8/1982, de 10 de agosto, de Estatuto de Autonomía de Aragón, modificada por la Ley Orgánica 6/1994, de 24 de marzo y por la Ley Orgánica 5/1996, de 30 de diciembre».

23 Publicado en el Boletín Oficial de las Cortes Generales, serie B n. ${ }^{\circ} 246-1$, de 12 de mayo de 2006.

24 Publicado en el Boletín Oficial de las Cortes Generales, serie B n. ${ }^{\circ} 253-1$, de 8 de septiembre de 2006. 
Tras la aprobación de este nuevo Estatuto, se emitió el Dictamen 61/07, por el Consejo Consultivo de La Rioja, sobre la posible inconstitucionalidad de algunos preceptos del nuevo Estatuto de Autonomía de Aragón, aprobado por Ley Orgánica 5/2007, de 20 de abril, de "reforma" del Estatuto de Autonomía de Aragón. No obstante, entre los eventuales motivos de inconstitucionalidad estudiados no se consideró el vicio en el procedimiento al tramitarse como "reforma" lo que era un nuevo Estatuto.

III.1.D. La interesada corrupción de la reforma valenciana.

La Comunidad Valenciana inició un auténtico proceso de reforma estatutaria. Basta leer la propuesta aprobada por el Parlamento valenciano para comprobarlo. Resultó, por tanto, correcto, el acuerdo de 12 de julio de 2005 de la Mesa del Congreso de los Diputados calificando la iniciativa como "propuesta de reforma de Estatuto de Autonomía ${ }^{25}$.

Ahora bien, en el proceso de discusión del texto en el Congreso de los Diputados, se produjo un cambio importante y especialmente significativo. El informe de la Ponencia ${ }^{26}$, en el Congreso de los Diputados, añadió un artículo 93 a la proposición de reforma con este tenor:

Artículo 93.

Se añade la siguiente disposición derogatoria:

"Disposición Derogatoria.

Queda derogada la Ley Orgánica 5/1982, de 1 de julio, de Estatuto de Autonomía de la Comunitat Valenciana, así como la Ley Orgánica 4/1991, de 13 de marzo, y la Ley Orgánica 5/1994, de 24 de marzo".

El añadido es especialmente significativo si se tiene en cuenta que no se presentó ninguna enmienda solicitando la introducción de este texto ${ }^{27}$. Ahora bien, la Ponencia estudia la proposición los días 13, 15 y 20 de diciembre de 2006. Y la fecha es muy importante porque se da la circunstancia de que antes, el 18 de octubre de 2005, la Mesa del Congreso había decidido calificar como "propuesta de reforma del Estatuto de autonomía", lo que era en realidad la propuesta de un nuevo Estatuto de autonomía para Cataluña.

A la vista de estas circunstancias se constata que el cambio introducido por la Ponencia no era inocente. Se trataba de evitar que la verdadera reforma valenciana dejara en evidencia la falsa reforma catalana. Y la forma de ocultar el fraude constitucional que se perpetraba con el Estatuto catalán fue corromper la iniciativa de verdadera reforma emprendida en Valencia. Y la

25 El acuerdo de la Mesa y el texto de la Propuesta de Reforma se publicaron en el Boletín Oficial de las Cortes Generales, serie B n. ${ }^{\circ}$ 196-1, de 15 de julio de 2005.

26 Los miembros de la Ponencia eran: Ramón Jáuregui Atondo, don Cipriá Císcar Casabán, don Elviro Aranda Alvarez (GS); don Federico Trillo-Figueroa Martínez-Conde, don Jaime Ignacio del Burgo Tajadura (GP); don Josep Antoni Duran i Lleida (GC-CiU), don Agustí Cerdá i Argent (GER-ERC), don Aitor Esteban Bravo (GV-EAJ-PNV), doña Isaura Navarro Casillas (GIV-IU-ICV), don Paulino Rivero Baute (GCC-NC) y doña Begoña Lasagabaster Olazábal (GMx).

27 Así se puede constatar en el el Boletín Oficial de las Cortes Generales, serie B n. ${ }^{\circ}$ 196-7, de 16 de noviembre de 2006 
forma de hacerlo fue la introducción de una disposición derogatoria del Estatuto vigente, como la que tenían el llamado "plan Ibarreche" y la propuesta de nuevo Estatuto catalán y que no fueron óbice para que la Mesa del Congreso calificara el texto como "reforma del Estatuto de autonomía". Es significativo que 8 de los 11 miembros de la Ponencia que introdujo esta disposición derogatoria fueran miembros de partidos que previamente habían promovido iniciativas que fueron calificadas fraudulentamente como "reformas" ${ }^{28}$.

Se puede decir, y es cierto, que también se presentaron como auténticas reformas estatutarias las de los Estatutos de Baleares y Castilla-León. Pero no es menos cierto, que cuando estos textos se presentan ante el Congreso de los Diputados (30 de junio de 2006 la reforma balear y 15 de diciembre de 2006, la reforma castellano-leonesa) el proceso catalán, que es el que verdaderamente quería sacar adelante la mayoría parlamentaria, ya estaba prácticamente concluido. En efecto, aunque la Ley Orgánica que aprueba el nuevo Estatuto catalán es de 17 de julio de 2006, la aprobación por el Senado se había hecho en mayo de 2006 y el referéndum sobre el mismo ya se había celebrado el 18 de junio de ese año.

Por tanto, mientras se estaba tramitando el nuevo Estatuto catalán, el único texto que podía dejar en evidencia que el mismo no era una auténtica "reforma" era la propuesta valenciana. De ahí el interés de los que apoyaron las propuestas fraudulentas que eran el "plan Ibarreche" y el nuevo Estatuto catalán en neutralizar un texto que pudiera poner de relieve el fraude constitucional que se estaba operando.

La corrupción de la reforma valenciana ha tenido un efecto esperpéntico. La reforma se aprueba con el título de "Ley Orgánica 1/2006, de 10 de abril de Reforma de la Ley Orgánica 5/1982, de 1 de julio, de Estatuto de Autonomía de la Comunidad Valenciana", pero resulta que en su disposición derogatoria deroga la ley que en su título reforma, a saber, la Ley Orgánica 5/1982.

III.1.E. La inconstitucionalidad de la derogación novativa de los Estatutos vigentes.

El problema de la distinción entre "derogación" o "reforma" no es baladí. ¿Por qué insisto tanto en distinguir entre "reforma" y "derogación" de un Estatuto? ${ }^{29}$ Por una razón muy sencilla: porque la Constitución, por razones varias, sólo permite a un parlamento autonómico promover la "reforma", pero no la "derogación" del Estatuto de autonomía. Los artículos 147.3 y 152.2 de la Constitución no dejan resquicio a ninguna duda.

28 El "Plan Ibarreche" fue promovido por PNV, EA e IU que contaban con 3 miembros en la Ponencia (Esteban, Navarro y Lasagabaster). El nuevo Estatuto catalán fue promovido por PSOE, ERC y CiU que contaban con cinco miembros en la Ponencia (Jáuregui, Císcar, Aranda, Durán y Cerdá).

29 He insistido ya en este punto en "El Estatuto de Galicia: ¿reforma? ¿necesaria?", en Vicente GARRIDO MAYOL (dtor.), Modelo de Estado y reforma de los Estatutos, Fundación Profesor Manuel Broseta/Corts Valencianes, Valencia, 2007, pág. 351 ss. (353-355). 
El art. 147.3 CE dice que:

"la reforma de los Estatutos se ajustará al procedimiento establecido en los mismos y requerirá, en todo caso, la aprobación por las Cortes Generales, mediante ley orgánica».

El art. 152.2 CE por su parte, establece que:

"una vez sancionados y promulgados los respectivos Estatutos, solamente podrán ser modificados mediante los procedimientos en ellos establecidos y con referéndum entre los electores inscritos en los censos correspondientes".

Por su parte, el Reglamento del Congreso de los Diputados, escuetamente, dispone en su art. 145 que :

"la reforma del Estatuto de autonomía tramitada conforme a las normas en el mismo establecidas, requerirá aprobación mediante Ley Orgánica”.

Para mí es evidente que cuando el art. 152.2 CE dice que "los respectivos Estatutos, solamente podrán ser modificados...", lo que está haciendo es tratar la misma cuestión que el art. 147.3 CE reserva a los estatutos de "vía lenta". No cabe duda, a mi juicio, que esta "modificación" debe entenderse como "reforma".

Se pueden indagar o discutir las razones de política constitucional que llevaron a establecer este precepto, pero el sentido del mismo es inequívoco. La Constitución de 1978 en ocasiones ha favorecido a las Comunidades Autónomas, como cuando, a diferencia de la Constitución de 1931 no contempla la posibilidad de que una provincia renuncie a formar parte de una comunidad autónoma. Pero también las impone límites. Y uno de ellos es, precisamente, el impedirlas promover un nuevo Estatuto. La razón no es difícil de alcanzar. Se trata de evitar, justamente, lo que acabamos de ver: a saber, que una autonomía con veleidades independentistas pueda promover una iniciativa que quiebre la unidad nacional.

No admite duda que un Estatuto aprobado puede ser reformado. Pero cuestión distinta es la de si las normas que prevén el procedimiento para hacer el Estatuto en un primer momento (las de los artículos 143.1 y 152.2 $\mathrm{CE}$ ), se podrían utilizar para hacer un "nuevo" Estatuto. La cuestión que se plantearía es la siguiente: ¿es posible aprobar abora un nuevo Estatuto de autonomía que derogue uno anterior?

La lectura de la Constitución, complementada con la del Reglamento del Congreso de los Diputados sólo ofrece dos respuestas.

a) La primera respuesta sería que estos preceptos constitucionales que regulan el modo de aprobar un Estatuto de autonomía son normas de "Derecho transitorio" que ya han sido agotadas por su uso. La consecuencia sería que, una vez aprobados los Estatutos, la Constitución 
sólo permite su reforma, pero en ningún caso la aprobación de un nuevo Estatuto.

b) La segunda respuesta sería que las normas constitucionales sobre aprobación del Estatuto de autonomía no serían un Derecho transitorio agotado. En consecuencia, la Constitución no impediría la aprobación de un nuevo Estatuto, pero eso sería posible únicamente respetando el procedimiento que la Constitución establece para la aprobación de un Estatuto ex novo: el fijado en los arts. 143 y 152 CE. Ese procedimiento, hay que recordarlo de nuevo, exige que el proyecto de Estatuto haya sido elaborado por una asamblea compuesta por los diputados y senadores de los territorios que pretenden el autogobierno, no por un parlamento autonómico.

Está, por tanto, absolutamente claro en la Constitución que un Parlamento autonómico carece por completo de legitimación para aprobar un nuevo Estatuto de autonomía. Precisamente esa imposibilidad constitucional explicaría la insistencia en calificar como "reforma estatutaria" lo que no es sino la aprobación de un "nuevo" Estatuto. Se trataría de sortear la inconstitucionalidad formal radical de la que adolecen las proposiciones presentadas por los parlamentos vasco y catalán, primero y andaluz y aragonés, después.

\section{III.2. NUEVOS REFERÉNDUMS CONVOCADOS INCONSTITUCIONALMENTE:}

\section{Cataluña y Andalucía}

III.2.A. Los hechos.

Después de que las Cortes Generales apoyaran la aprobación de las pseudo-reformas de los Estatutos catalán y andaluz, dichos textos fueron sometidos a referéndum. No fue el caso del Estatuto aragonés.

III.2.A.a. La convocatoria del referéndum sobre el Estatuto catalán se realizó mediante el Decreto 170/2006, de 18 de mayo por el que se somete a referéndum el proyecto de reforma del Estatuto de autonomía de Cataluña ${ }^{30}$. Este Decreto está firmado, únicamente, por el Presidente de la Generalidad de Cataluña. La Exposición de Motivos de este Decreto dice lo siguiente:

"El artículo 56 del Estatuto de autonomía de Cataluña dispone que una vez aprobado el proyecto de reforma por las Cortes Generales mediante ley orgánica se debe proceder a someterlo a referéndum positivo de las personas electoras de Cataluña, por lo cual corresponde convocarlo mediante este Decreto.

En conformidad con la habilitación legal mencionada y de acuerdo con el artículo 60 de la Ley 3/1982, de 23 de marzo, del Parlamento, del Presidente y del Consejo Ejecutivo de la Generalidad, decreto: (...)

Barcelona, 18 de mayo de 2006.-El Presidente, Pasqual Maragall i Mira.

30 Diario Oficial de la Generalidad de Cataluña n. ${ }^{\circ 637}$, de 19 de mayo, páginas 22006 y siguientes; y Boletín Oficial del Estado de 19 de mayo, páginas 19072 y siguientes. 
Adviértase de tres circunstancias: a) El Decreto habla de "reforma" del Estatuto; b) el Decreto presume que el objeto del referéndum es una «ley orgánica»; y c) está firmado por "El Presidente" de la Generalidad de Cataluña. Todas estas circunstancias, como veremos, son muy relevantes para determinar la ilegalidad e inconstitucionalidad de este Decreto.

III.2.A.b. Por lo que hace al Estatuto andaluz, el referéndum sobre el mismo se convocó mediante Decreto del Presidente (de la Comunidad Autónoma) 2/2007, de 16 de enero, por el que se somete a referéndum el Proyecto de Reforma del Estatuto de Autonomía para Andalucía. La Exposición de Motivos de este Decreto, ligeramente diferente del Decreto catalán, es del siguiente tenor:

"El artículo 74.1.b) del Estatuto de Autonomía para Andalucía dispone que la propuesta de reforma del Estatuto requerirá, en todo caso, la aprobación del Parlamento Andaluz por mayoría de tres quintos, la aprobación de las Cortes Generales mediante Ley Orgánica y, finalmente, el referéndum positivo de las personas electoras de Andalucía.

El día 20 de diciembre de 2006 las Cortes Generales han aprobado la Ley Orgánica de reforma del Estatuto de Autonomía para Andalucía, cuya Disposición Adicional Quinta señala que una vez aprobada la ley orgánica de reforma de dicho Estatuto, el Gobierno de la Nación deberá autorizar la convocatoria del referéndum previsto en el artículo 74.1.b) de la Ley Orgánica 6/1981 en el plazo de seis meses.

El Gobierno de la Nación ha autorizado la convocatoria del citado referéndum mediante acuerdo del Consejo de Ministros del día 22 de diciembre de 2006, por lo cual, cumplidas todas las previsiones legales, corresponde convocarlo mediante este Decreto.

En su virtud, de conformidad con la habilitación legal mencionada y con lo establecido en el artículo 2 de la Ley 6/2006, de 24 de octubre, del Gobierno de la Comunidad

Autónoma de Andalucía, dispongo: (...)

Sevilla, 16 de enero de 2007.-El Presidente, Manuel Chaves González.»

En el Decreto andaluz conviene advertir las siguientes circunstancias: a) El Decreto habla de "reforma" del Estatuto; b) el Decreto presume que el objeto del referéndum es una "ley orgánica»; c) está firmado por "El Presidente" de la Comunidad Autónoma; d) Se dice que el Gobierno de la Nación ha autorizado la convocatoria del citado referéndum.

III.2.B. La ambigüedad normativa no oculta la radical inconstitucionalidad de la convocatoria de los referéndums.

III.2.B.a. La Constitución establece en su artículo 149.1.32. ${ }^{\circ}$ que el Estado tiene competencia exclusiva para dar la «autorización para la convocatoria de consultas populares por vía de referéndum". Esos referenda deben ser convocados por el rey, pues el art. 62.c) le atribuye "convocar a referéndum en los casos previstos por la Constitución". De esos casos habla el art. 92.3 CE que dice que "Una Ley orgánica regulará las condiciones y el procedimiento de las distintas modalidades de referéndum previstas en esta Constitución". La 
previsión de los arts. 62.c) y 92.3 CE se desarrolló en la la LO 2/1980 sobre regulación de las distintas modalidades de referéndum. Entre esas modalidades incluye (art. 10) el referéndum de "modificación" de Estatutos previsto en el art.152.2 CE. A todos los referenda contemplados en la LO 2/1980, incluido, por tanto, el de "modificación" de Estatutos de vía rápida, son de aplicación las previsiones de los arts. 1 y 2 de la LO 2/1980.

El art. 1 de la LO 2/1980 dispone que:

"El referéndum en sus distintas modalidades, se celebrará de acuerdo con las condiciones y procedimientos regulados en la presente Ley Orgánica».

Y el art. 2 reza:

"Uno. La autorización para la convocatoria de consultas populares por vía de referéndum en cualquiera de sus modalidades, es competencia exclusiva del Estado.

Dos. La autorización será acordada por el Gobierno, a propuesta de su Presidente, salvo en el caso en que este reservada por la Constitución al Congreso de los Diputados.

Tres. Corresponde al Rey convocar a referéndum, mediante Real Decreto acordado en Consejo de Ministros y refrendado por su Presidente».

III.2.B.b. El primer problema que se plante es el de si el referéndum de reforma de los Estatutos de "Vía rápida" previsto en el art. 152.2 CE podría estar regulado en otra ley orgánica distinta, como es el Estatuto de Autonomía. De hecho, tanto el Estatuto catalán como el andaluz contienen previsiones similares entre sí (el art. 56.1 de la vigente Ley Orgánica 4/1979, que aprueba el Estatuto de Autonomía de Cataluña; art. 74.1.b de la Ley Orgánica 6/1981, que aprueba el Estatuto de Autonomía de Andalucía) que se separan de lo que dispone la LO /1980. En ambos Estatutos se establece que

"la aprobación de la reforma (del Estatuto de autonomía) por las Cortes Generales mediante Ley Orgánica incluirá la autorización del Estado para que la Generalidad convoque el referéndum».

A nuestro juicio, sin embargo, estos preceptos estatutarios son inconstitucionales. El Estatuto de Autonomía es una ley orgánica especial que, dadas las dificultades de su aprobación, reforma y derogación no puede incluir otros contenidos distintos de los expresamente previstos en la Constitución. El artículo 92 de la Constitución establece, claramente, que la regulación de todos los referendums tiene que hacerse por una ley orgánica del Estado, una ley orgánica "normal".

En el caso más extremo, sólo podría darse una interpretación conforme a la Constitución del precepto del Estatuto catalán (que no del andaluz) si se estima que en aquella fecha aún no se había dictado la ley orgánica que regulaba las distintas modalidades de referéndum y que, por ello, pretendía 
cubrir una laguna jurídica. Sin embargo, desde que se dictó la ley orgánica 2/1980, la regulación sobre el referéndum del Estatuto catalán ha devenido inaplicable por invadir una competencia exclusiva del Estado.

En consecuencia, ante la existencia de estas leyes orgánicas divergentes, debe aplicarse la ley orgánica 2/1980 por ser ésta la que es plenamente conforme con la Constitución.

III.2.B.c. Si, como creemos, la regulación constitucionalmente procedente para la celebración de los referenda de modificación de un Estatuto es una competencia exclusiva del Estado que debe ejercerse según lo dispuesto en la Ley Orgánica 2/1980, el resultado es que el referéndum convocado sobre el Estatuto catalán ha violado el art. 149.1.32 CE y el referéndum sobre el Estatuto catalán y sobre el Estatuto andaluz, lo dispuesto en los arts. 62.c) 92.3 CE.

El primer motivo de inconstitucionalidad (que afecta al referéndum catalán) es claro. El presidente de la Generalidad de Cataluña, Pasqual Maragall, convocó, por su cuenta, un referéndum sin tener ninguna autorización del Estado. El texto del Decreto 170/2006 no deja lugar a ninguna duda.

A este respecto, resulta enormemente ilustrativo recordar que en la VIII Legislatura (2004-2008), la misma en la que se aprobó el nuevo Estatuto catalán, se presentaron dos proposiciones de ley para modificar la Ley Orgánica 2/1980, que pretendían permitir lo que luego hizo el Presidente de la Generalidad. Ambas fueron rechazadas en el debate de toma en consideración ${ }^{31}$. Además, después de aprobado el nuevo Estatuto se intentó, nuevamente, atribuir a la Generalidad la competencia de convocatoria de referenda ${ }^{32}$.

El segundo motivo de inconstitucionalidad (que afecta a los referenda en Cataluña y Andalucía) no es menos claro que el primero. No hay duda de que, de acuerdo con lo dispuesto en los arts. 1 y 2 de la LO 2/1980 esos referéndums debían ser convocados por el Rey mediante un Decreto aprobado en Consejo de Ministros y refrendado por su presidente. En el caso de Cataluña es evidente que no hay intervención ninguna del Consejo de Ministros.

31 Proposición de Ley Orgánica, por vía del artículo 150.2 de la Constitución, de transferencia a la Generalidad de Cataluña de las competencias en materia de autorización para la convocatoria de referéndum (corresponde al número de expediente 125/000040/0000 de la VII Legislatura). (125/000012), presentada por el Parlamento de Cataluña (publicada en el Boletín Oficial de las Cortes Generales, n. ${ }^{\circ}$ B-12-1 de 16 de abril de 2004) y cuya toma en consideración fue rechazada (Boletín Oficial de las Cortes Generales, n. ${ }^{\circ}$ B-12-2 de 13 de febrero de 2006).

Proposición de Ley de transferencia a las Comunidades Autónomas por la vía del artículo 150.2 de la Constitución, de las competencias en materia de autorización para la convocatoria de referéndum (Orgánica). (122/000079), presentada por el Grupo Parlamentario de Esquerra Republicana (ERC) (publicada en el Boletín Oficial de las Cortes Generales, n. ${ }^{\circ}$ B-95-1 de 12 de julio de 2004) y cuya toma en consideración fue rechazada (Boletín Oficial de las Cortes Generales, n. ${ }^{\circ}$ B-95-2 de 2 de abril de 2007).

32 Proposición de Ley Orgánica de transferencia a la Generalitat de Cataluña de las facultades de ejecución de la legislación del Estado para la autorización de la convocatoria de consultas populares por vía de referéndum. (122/000211) presentada por el Grupo Parlamentario de Esquerra Republicana (ERC) (publicada en el Boletín Oficial de las Cortes Generales, n. ${ }^{\circ}$ B-266-1 de 27 de octubre de 2006). Esta iniciativa caducó con la disolución de la Cámara. 
En el caso de Andalucía sí que hay una intervención del Consejo de Ministros, pero ilegal. Y es ilegal porque aunque se habla de un "acuerdo del Consejo de Ministros" autorizando la convocatoria del referéndum, esa autorización no puede ser sino para que lo convoque el Rey, no el presidente de la Comunidad autónoma. El art. 2.3 de la LO 2/1980 no admite margen de discusión.

III.2.B.d. Pero la convocatoria de los referenda catalán y andaluz, junto a la inconstitucionalidad antes señalada, merece una tacha de ilegalidad por otros motivos adicionales. Incluso en el supuesto de que se considerara que lo aplicable era el Estatuto de autonomía de Cataluña o de Andalucía (y no la LO 2/1980), aquí se argumenta que los referenda convocados mediante el Decreto de la Generalidad 170/2006, de 18 de mayo y el Decreto del Presidente de la Junta de Andalucía 2/2007 incurren en una doble ilegalidad.

En primer lugar, ambos referéndums son ilegales porque no se da el primer supuesto de hecho habilitante para la convocatoria del referéndum, que exige que lo aprobado sea una reforma. En efecto, aunque los títulos de los Decretos digan que se somete a referéndum el "proyecto de reforma del Estatuto", los anexos de ambos Decretos reproducen íntegramente el texto de sendos nuevos Estatutos, no de los Estatutos anteriores reformados. Nuevos Estatutos que derogan los anteriores como se desprende, inequívocamente, del hecho de que contienen sendas disposiciones derogatorias que dicen, textualmente: "Queda derogada la Ley Orgánica 4/1979, de 18 de diciembre, de Estatuto de Autonomía de Cataluña" y "Queda derogada la Ley Orgánica 6/1981, de 30 de diciembre, de Estatuto de Autonomía para Andalucia.. Si los Estatutos catalán de 1979 y andaluz de 1981 quedan derogados es, evidentemente, porque no son reformados sino sustituidos por otros nuevos.

En segundo lugar, los referéndums son ilegales porque no se da el segundo supuesto de hecho habilitante para la convocatoria del referéndum, que es el que se halla aprobado una reforma mediante "ley orgánica". Para que exista una ley, sea ordinaria u orgánica se exige, de acuerdo con el artículo 91 de la Constitución que el texto aprobado por las Cortes Generales sea sancionado y promulgado por el rey, "en el plazo de quince dias". Mientras un texto no contenga la oportuna sanción y promulgación puede ser denominado de cualquier manera, pero no como «ley». En efecto, sólo la ley sancionada y promulgada pertenece al ordenamiento jurídico y, por tanto, confiere facultades, otorga derechos e impone obligaciones. Es evidente que ni el nuevo Estatuto de autonomía de Cataluña ni el nuevo Estatuto andaluz había sido ni sancionado, ni promulgado cuando fue sometido a referéndum. Por tanto sólo era una proposición legislativa, pero nunca una ley. Si el nuevo Estatuto no era aún una ley orgánica cuando se sometió a referéndum, este referéndum no podría convocarse, si nos atenemos a la literalidad del art. 56.3 del Estatuto de Cataluña actualmente vigente. 


\section{IV.CONCLUSIÓN}

El proceso autonómico en España se ha articulado en dos etapas: la primera, inmediatamente después de operada la transición desde el franquismo; la segunda, articulada en la llamada "segunda transición" pilotada con el acceso al poder de Rodríguez Zapatero. En ambas etapas, el proceso autonómico se halla aquejado de vicios de origen que afectan severamente a su legitimidad en un Estado que se define como "de Derecho" y que proclama la obligación de que los poderes públicos actúen respetando el marco constitucional y su desarrollo legal.

En el primer proceso autonómico, las cuatro regiones que accedieron al tipo de autonomía de "vía rápida" lo hicieron violando diversas disposiciones constitucionales.

Los Estatutos de las dos primeras Comunidades Autónomas (País Vasco y Cataluña) fueron sometidos a un referéndum convocado con infracción de lo dispuesto en el artículo 92.3 de la Constitución.

Para Galicia se tramitó un Estatuto que no fue el que se aprobó por la Comisión Constitucional teniendo en cuenta la opinión de la Asamblea de Parlamentarios, infringiendo lo dispuesto en el artículo 151.2.2 de la Constitución.

Por su parte, en Andalucía el Estatuto de autonomía fue el resultado de una iniciativa que no fue apoyada por la mayoría absoluta de los electores de la provincia de Almería, violando lo que establece el artículo 151.1 de la Constitución.

El segundo proceso autonómico se desencadena después de que la Mesa del Congreso calificara fraudulentamente como "reforma estatutaria" una iniciativa, el llamado "Plan Ibarreche» que no era tal. A partir de ahí, se tramitaron como "reformas estatutarias" los nuevos Estatutos de Cataluña, Andalucía y Aragón que derogaban los Estatutos hasta entonces vigentes, violando lo dispuesto en los artículos 152.2 y 147.3 de la Constitución que sólo permiten a las Asambleas parlamentarias autonómicas tramitar "reformas" de los Estatutos, pero no iniciar un nuevo Estatuto.

A mayor abundancia, en los procesos que condujeron a la aprobación de los nuevos Estatutos de Cataluña y de Andalucía se celebraron sendos referenda que, habiendo sido convocados por los respectivos presidentes autonómicos, violaron así lo dispuesto en los artículos 62.c), 149.1.32 y 92.3 de la Constitución que establecen la competencia del Estado para autorizar estas consultas que deben ser convocadas por el Rey.

TitLE Original vices in the autonomy processes.

AвSTRACT: The more important processes in Spain to grant regional autonomy suffer from original vices which consist in the violation of the constitutional provisions concerning its draft and reform. The first process to grant autonomy, which gave birth to the Autonomous regions 
based upon the article 151 of the Constitution, presents several original vices, wether the statutes were processed taking into account referendums bold inconstitutionally, or because relevant provisions of the parliamentary process were omitted. The second process to grant autonomy, which broke out since 2003, is articulated through suppossed "reforms" of the Autonomy Statutes which really are abrogations of them passing new Autonomy Statutes as well as through referendums hold again on violation of relevant constitutional and legal provisions.

Resumen: Los más importantes procesos estatutarios en España se hallan aquejados de vicios en su origen consistentes en la violación de las prescripciones constitucionales sobre su elaboración y reforma. El primer proceso autonómico, que dio lugar a todas las Comunidades autónomas creadas al amparo del artículo 151 de la Constitución presenta diversos vicios de origen, bien porque se tramitaron con referendums celebrados inconstitucionalmente, o porque se concedió validez a referendums que no la tenían o porque se omitieron las reglas sobre su tramitación parlamentaria. El segundo proceso autonómico, desencadenado a partir de 2003, por su parte, se articula sobre supuestas "reformas" estatutarias que no son sino derogaciones novativas de los Estatutos anteriores, y sobre referéndums nuevamente convocados en violación de la Constitución y la ley.

KeY WORDs: Autonomy Statute. Autonomy statute procedure. Reform. Abrogation. Referendum.

Palabras clave: Estatuto de autonomía. Proceso estatutario. Reforma. Derogación. Referéndum. 